\title{
@creative
}

ISSN 2590-9770

The Art of Discrete and Applied Mathematics 4 (2021) \#P1.01

https://doi.org/10.26493/2590-9770.1312.5b4

(Also available at http://adam-journal.eu)

\section{Palindromic products*}

\author{
Richard H. Hammack ${ }^{\dagger}$ (D), Jamie L. Shive \\ Virginia Commonwealth University, Dept. of Mathematics, Richmond, VA 23284, USA
}

Received 28 July 2019, accepted 20 January 2020, published online 8 September 2020

\begin{abstract}
A graph $G$ on $n$ vertices is said to be palindromic if there is a vertex-labeling bijection $f: V(G) \rightarrow\{1,2, \ldots, n\}$ with the property that for any edge $v w \in E(G)$ there is an edge $x y \in E(G)$ for which $f(x)=n-f(v)+1$ and $f(y)=n-f(w)+1$.

This notion was defined and explored in a recent paper [R. Beeler, Palindromic graphs, Bulletin of the ICA, 85 (2019) 85-100]. The paper gives sufficient conditions on the factors of a Cartesian product of graphs that ensure the product is palindromic, but states that it is unknown whether the conditions are necessary. We prove that the conditions are indeed necessary. Further, we prove a parallel result for the strong product of graphs.
\end{abstract}

Keywords: Palindromic graphs, cartesian product of graphs, strong product of graphs.

Math. Subj. Class. (2020): 05C76, 05C78

\section{Introduction}

A recent article by R. Beeler [1] introduced a new concept. A graph $G$ on $n$ vertices is palindromic provided that there is a vertex-labeling bijection $f: V(G) \rightarrow\{1,2, \ldots, n\}$ with the property that to each $v w \in E(G)$ there corresponds an $x y \in E(G)$ for which $f(x)=n+1-f(v)$ and $f(y)=n+1-f(w)$.

Palindromic graphs, like palindromic words, have a certain symmetry. The mapping $V(G) \rightarrow V(G)$ whose effect on labels is $k \mapsto n+1-k$ is an involution (an automorphism of order 2). View it as a mirror symmetry, where the vertices are ordered on a line by their labels, as in Figure 1.

This induced involution has no fixed vertex if $n$ is even, and exactly one fixed vertex if $n$ is odd. Indeed, we have the following characterization of palindromic graphs as those graphs admitting an involution that fixes at most one vertex. (The order of a graph is its number of vertices. For other standard terms and notations not defined here see West [5].)

\footnotetext{
* We thank the referees.

${ }^{\dagger}$ Supported by Simons Foundation Collaboration Grant for Mathematicians 523748.

E-mail addresses: rhammack@vcu.edu (Richard H. Hammack), shivejl@vcu.edu (Jamie L. Shive)
} 


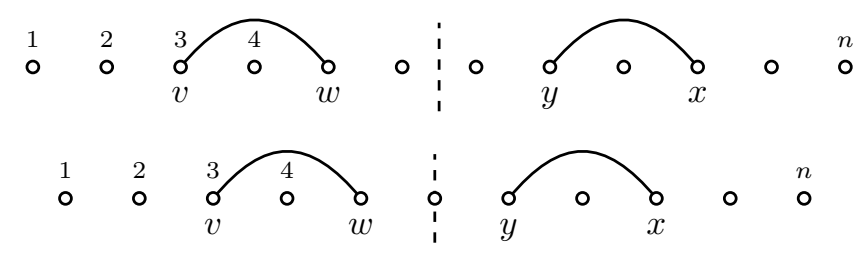

Figure 1: Palindromic graphs of even order admit an involution with no fixed points. Palindromic graphs of odd order admit an involution with exactly one fixed point.

Theorem 1.1 (Beeler [1]). A graph of even order is palindromic if and only if it admits an involution with no fixed vertices. A graph of odd order is palindromic if and only if it admits an involution with exactly one fixed vertex.

Guided by this theorem, we define a graph to be even palindromic if it is palindromic and of even order; it is odd palindromic if it is palindromic and of odd order. An involution that fixes at most one vertex is called a palindromic involution; one that fixes no vertex is an even palindromic involution, and one that fixes exactly one vertex is an odd palindromic involution. Thus a graph is even palindromic if and only if admits an even palindromic involution; it is odd palindromic if and only if it admits an odd palindromic involution. A fixed point is a fixed vertex.

Beeler [1] characterizes several classes of palindromic graphs, including hypercubes (see Figure 2). More generally he addresses the Cartesian product of graphs, and we will expand upon this in the next section.

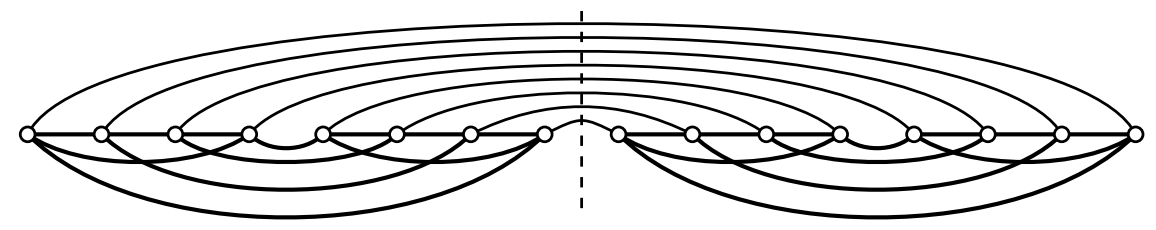

Figure 2: Every hypercube is palindromic. Here is the 4-cube.

\section{Cartesian Products}

The Cartesian product of graphs $G$ and $H$ is the graph $G \square H$ with vertices $V(G) \times V(H)$ and edges

$$
E(G \square H)=\left\{(x, y)\left(x^{\prime}, y^{\prime}\right) \mid x x^{\prime} \in E(G) \text { and } y=y^{\prime} \text {, or } x=x^{\prime} \text { and } y y^{\prime} \in E(H)\right\} .
$$

(See Figure 3.) This product is commutative and associative in the sense that the maps $(x, y) \mapsto(y, x)$ and $((x, y), z) \mapsto(x,(y, z))$ are isomorphisms $G \square H \rightarrow H \square G$ and $(G \square H) \square K \rightarrow G \square(H \square K)$.

Given automorphisms $\alpha: G \rightarrow G$ and $\beta: H \rightarrow H$, it is straightforward from the definitions that $(x, y) \mapsto(\alpha(x), \beta(y))$ is an automorphism of $G \square H$. For example, in Figure 3, let $\alpha: G \rightarrow G$ be the even palindromic involution of $G$ reflecting $G$ across 


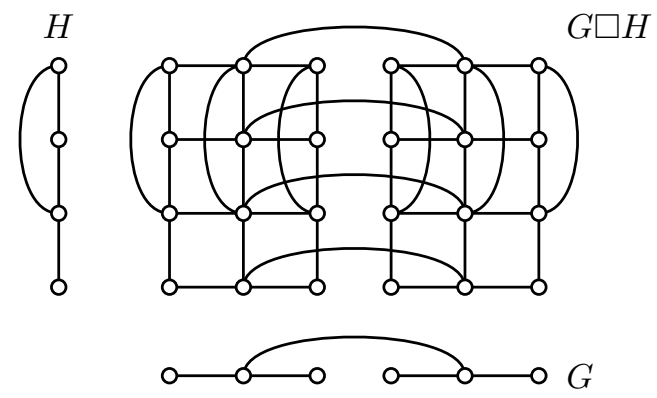

Figure 3: Cartesian product of graphs.

a vertical axis. Let $\beta: H \rightarrow H$ be the identity. Then $(x, y) \mapsto(\alpha(x), y)$ is an even palindromic involution of $G \square H$ reflecting it across a vertical axis. This suggests that if one factor of a product is even palindromic, then the product will be even palindromic. Indeed, we have the following result [1, Theorem 4.4].

Lemma 2.1. If $G$ or $H$ is even palindromic, then $G \square H$ is even palindromic. If $G$ and $H$ are odd palindromic, then $G \square H$ is odd palindromic.

Proof. Let one of $G$ or $H$ (say $G$ ) be even palindromic. Theorem 1.1 yields an even palindromic involution $\alpha: G \rightarrow G$. Form the even palindromic involution $(x, y) \mapsto$ $(\alpha(x), y)$ of $G \square H$. Thus the product is even palindromic. For the second statement, say both $G$ and $H$ are odd palindromic. By Theorem 1.1, $G$ has an involution $\alpha$ with exactly one fixed point $x_{0}$. (That is, $\alpha\left(x_{0}\right)=x_{0}$.) For the same reason, $H$ has an involution $\beta$ with exactly one fixed point $y_{0}$. Then $(x, y) \mapsto(\alpha(x), \beta(y))$ is an involution of $G \square H$ that has exactly one fixed point $\left(x_{0}, y_{0}\right)$. Therefore $G \square H$ is odd palindromic.

Lemma 2.1 spells out conditions on the factors that are sufficient for a palindromic product. Beeler [1] states that it is unknown whether these conditions are also necessary. We will shortly prove that in fact they are, but we first need to review prime factorizations over the Cartesian product.

Observe that $K_{1} \square G \cong G$ for any graph $G$, so $K_{1}$ is the unit for the Cartesian product. A nontrivial graph $G$ is prime over $\square$ if for any factoring $G \cong A \square B$, one of $A$ or $B$ is $K_{1}$ and the other is isomorphic to $G$. Certainly every graph can be factored into prime factors. Sabidussi and Vizing [3, 4] proved that each connected graph has a unique prime factoring up to order and isomorphism of the factors. More precisely, we have the following.

Theorem 2.2 ([2, Theorem 6.8]). Let $G$ and $H$ be isomorphic connected graphs $G=$ $G_{1} \square \cdots \square G_{k}$ and $H=H_{1} \square \cdots \square H_{\ell}$, where each factor $G_{i}$ and $H_{i}$ is prime. Then $k=\ell$, and for any isomorphism $\varphi: G \rightarrow H$, there is a permutation $\pi$ of $\{1,2, \ldots, k\}$ and isomorphisms $\varphi_{i}: G_{\pi(i)} \rightarrow H_{i}$ for which

$$
\varphi\left(x_{1}, x_{2}, \ldots, x_{k}\right)=\left(\varphi_{1}\left(x_{\pi(1)}\right), \varphi_{2}\left(x_{\pi(2)}\right), \ldots, \varphi_{k}\left(x_{\pi(k)}\right)\right) .
$$

Now we can prove our main result about palindromic Cartesian products.

Theorem 2.3. Suppose $G$ and $H$ are connected graphs. Then:

(1) $G$ or $H$ is even palindromic if and only if $G \square H$ is even palindromic. 
(2) $G$ and $H$ are odd palindromic if and only if $G \square H$ is odd palindromic.

Proof. One direction is Lemma 2.1. Conversely, suppose $G \square H$ is palindromic and let $\varphi$ be a palindromic involution of it. Take prime factorings $G=G_{1} \square \cdots \square G_{j}$ and $H=$ $G_{j+1} \square \cdots \square G_{k}$, so $\varphi$ is an involution of $G \square H=\left(G_{1} \square \cdots \square G_{j}\right) \square\left(G_{j+1} \square \cdots \square G_{k}\right)$.

The involution $\varphi$ permutes the prime factors of this product in the sense of Theorem 2.2, where the permutation $\pi$ satisfies $\pi^{2}=\mathrm{id}$. Using commutativity of $\square$, group together the prime factors $G_{i}$ of $G$ for which $1<\pi(i) \leq j$, and call their product $A$. (By convention, $A=K_{1}$ if there are no such factors $G_{i}$. The same applies for the graphs $B$ and $D$ defined below.) Let $B$ be the product of the remaining factors $G_{i}$ of $G$. Also group together the prime factors $G_{i}$ of $H$ for which $j+1<\pi(i) \leq k$, and call their product $D$. The Cartesian product of the remaining factors of $H$ is then a graph isomorphic to $B$. The structure of $\varphi$ under this scheme is as indicated below, where the arrows represent isomorphisms $\varphi_{i}: G_{\pi(i)} \rightarrow G_{i}$ between factors.

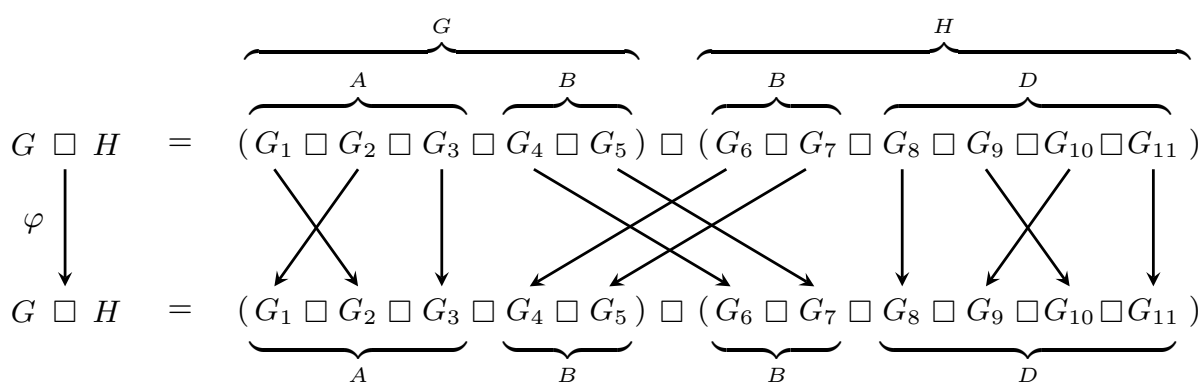

We have coordinatized $G$ and $H$ as $G=A \square B$ and $H=B \square D$, and $\varphi$ is an involution of $G \square H=(A \square B) \square(B \square D)$ for which $\varphi\left((a, b),\left(b^{\prime}, d\right)\right)=\left(\left(\alpha(a), \beta\left(b^{\prime}\right)\right),(\gamma(b), \delta(d))\right)$, for automorphisms $\alpha: A \rightarrow A, \beta, \gamma: B \rightarrow B$ and $\delta: D \rightarrow D$. But because $\varphi^{2}$ is the identity, it must be that $\alpha^{2}=\mathrm{id}, \gamma=\beta^{-1}$ and $\delta^{2}=\mathrm{id}$. Thus we have involutions $\alpha$ and $\delta$ of $A$ and $D$, respectively, and

$$
\varphi\left((a, b),\left(b^{\prime}, d\right)\right)=\left(\left(\alpha(a), \beta\left(b^{\prime}\right)\right),\left(\beta^{-1}(b), \delta(d)\right)\right),
$$

From (2.1) it is evident that the fixed points of $\varphi$ (if any) are precisely

$$
\left(\left(a_{0}, \beta(b)\right),\left(b, d_{0}\right)\right) \quad \text { with } \alpha\left(a_{0}\right)=a_{0}, \delta\left(d_{0}\right)=d_{0} \text {, and } b \in V(B) .
$$

Thus $\varphi$ has a fixed point if and only if both $\alpha$ and $\delta$ have fixed points. Further, if $\varphi$ has a fixed point, then it has exactly $|V(B)|$ of them.

Now suppose $G \square H$ is even palindromic. Let $\varphi$ be an even palindromic involution of $G \square H$ (having no fixed point). From (2.2), at least one of $\alpha$ or $\delta$ has no fixed point; say it is $\alpha$. Then $\alpha$ is an even palindromic involution of $A$, so $A$ is even palindromic. By the first part of the theorem, $G=A \square B$ is even palindromic. Similarly $H$ is even palindromic if $\delta$ has no fixed points.

Suppose $G \square H$ is odd palindromic. Let $\varphi$ be an odd palindromic involution whose sole fixed point is $\left(\left(a_{0}, \beta\left(b_{0}\right)\right),\left(b_{0}, d_{0}\right)\right)$. The remark following (2.2) implies $\varphi$ has at least $|V(B)|$ fixed points, so $B=K_{1}$. Thus we can drop $B$ from our discussion, so $G=A, H=D$ and $\varphi(a, d)=(\alpha(a), \delta(d))$. We now have involutions $\alpha: G \rightarrow G$ and $\delta: H \rightarrow H$ with fixed points $a_{0}$ and $d_{0}$, respectively. Also $\left(a_{0}, d_{0}\right)$ is a fixed point of $\varphi$. If 
the involution $\alpha$ of $G$ had a second fixed point $a_{1}$, then $\left(a_{0}, d_{0}\right)$ and $\left(a_{1}, d_{0}\right)$ would be two distinct fixed points of $\varphi$. Thus $a_{0}$ is the only fixed point of $\alpha$, so $\alpha$ (hence also $G$ ) is odd palindromic. By the same reasoning $H$ is odd palindromic.

\section{Strong Products}

The strong product of graphs $G$ and $H$ is the graph $G \otimes H$ with vertex set $V(G) \times V(H)$, where distinct vertices $(x, y)$ and $\left(x^{\prime}, y^{\prime}\right)$ are adjacent whenever

$$
\left(x x^{\prime} \in E(G) \text { or } y=y^{\prime}\right) \text { and }\left(x=x^{\prime} \text { or } y y^{\prime} \in E(H)\right) \text {. }
$$

See Figure 3. We quickly review this product's properties; Chapter 7 of [2] proves all assertions made here. The strong product is commutative and associative. If $N_{G}[x]:=$ $N(x) \cup\{x\}$ is the closed neighborhood of a vertex $x \in V(G)$, then

$$
N_{G \otimes H}[(x, y)]=N_{G}[x] \times N_{H}[y] .
$$

Also $K_{1} \otimes G \cong G$ for all graphs $G$. A graph $G$ is prime over $\otimes$ if for any factoring $G=A \otimes B$, one of $A$ or $B$ is $K_{1}$ and the other is isomorphic to $G$.

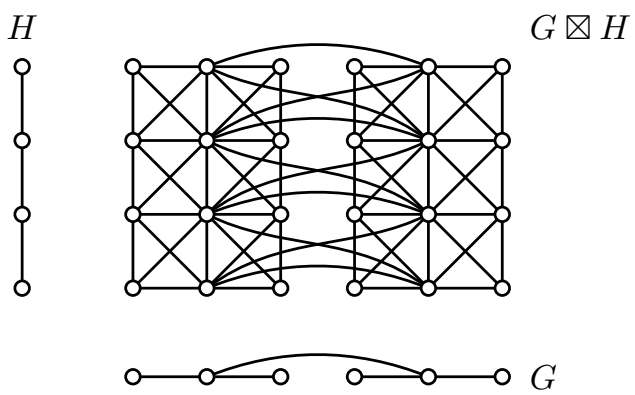

Figure 4: Strong product of graphs.

Given automorphisms $\alpha: G \rightarrow G$ and $\beta: H \rightarrow H$, it is straightforward from the definitions that $(x, y) \mapsto(\alpha(x), \beta(y))$ is an automorphism of $G \otimes H$. For instance, in Figure 4, let $\alpha: G \rightarrow G$ be the even palindromic involution of $G$ reflecting $G$ across a vertical axis. Say $\beta: H \rightarrow H$ is the identity. Then $(x, y) \mapsto(\alpha(x), y)$ is an even palindromic involution of $G \otimes H$ reflecting it across a vertical axis (relative to the drawing).

This suggests that we might expect a result for the strong product that is parallel to Theorem 2.3 for the Cartesian product. Indeed, this is exactly the case, but the proof is more involved. The complication is that in general the strong product has no result parallel to Theorem 2.2, unless we impose an additional restriction. A graph is called S-thin if no two distinct vertices have the same closed neighborhood. We will need the following analogue of Theorem 2.2 for $S$-thin graphs.

Theorem 3.1 ([2, Theorem 7.16]). Let $\varphi$ be an automorphism of an $S$-thin connected graph $G$ with prime factorization $G=G_{1} \otimes G_{2} \otimes \cdots \otimes G_{k}$. Then there is a permutation $\pi$ of $\{1,2, \ldots, k\}$ and isomorphisms $\varphi_{i}: G_{\pi(i)} \rightarrow G_{i}$ for which $\varphi\left(x_{1}, x_{2}, \ldots, x_{k}\right)=$ $\left(\varphi_{1}\left(x_{\pi(1)}\right), \varphi_{2}\left(x_{\pi(2)}\right), \ldots, \varphi_{k}\left(x_{\pi(k)}\right)\right)$. 
We say that vertices $x$ and $y$ of a graph are in relation $S$, written $x S y$, provided that each has the same closed neighborhood, that is, $N[x]=N[y]$. It is easy to check that $S$ is an equivalence relation of the graph's vertex set. We call an $S$-equivalence class of $V(G)$ an $\mathbf{S}$-class of $G$. (Note that a graph is $S$-thin if and only if each $S$-class consists of a single vertex.) In general, if $X$ is an $S$-class of graph $G$, then the subgraph of $G$ induced on $X$ is the complete graph $K_{|X|}$. Also, for any distinct $S$-classes $X$ and $Y$, either each vertex of $X$ is adjacent to all vertices of $Y$, or no vertex of $X$ is adjacent to any vertex of $Y$.

Given a graph $G$, we define the quotient $G / S$ to be the graph whose vertices are the $S$-classes of $G$, and for which $X Y \in E(G / S)$ provided that $X \neq Y$ and $G$ has an edge joining $X$ to $Y$. Check that $G / S$ is always $S$-thin.

Because $S$ is defined in terms of the adjaceny structure of a graph, any isomorphism $\varphi: G \rightarrow H$ sends $S$-classes of $G$ bijectively onto $S$-classes of $H$. From the discussion above it should be clear that any isomorphism $\varphi: G \rightarrow H$ induces an isomorphism $\widetilde{\varphi}$ : $G / S \rightarrow H / S$ where $\widetilde{\varphi}(X)=\varphi(X)$, that is, $\widetilde{\varphi}(X)$ is the image of the $S$-class $X$ under $\varphi$.

But the existence of an isomorphism $\widetilde{\varphi}: G / S \rightarrow H / S$ does not necessarily mean that there is an isomorphism $\varphi: G \rightarrow H$. However, if $|X|=|\widetilde{\varphi}(X)|$ for each $X \in V(G / S)$, then we can lift $\widetilde{\varphi}$ to an isomorphism $\varphi: G \rightarrow H$ simply by declaring $\varphi$ to restrict to a bijection $X \rightarrow \widetilde{\varphi}(X)$ for each $X$.

Using Equation (3.1), one can show that the $S$-classes of $G \otimes H$ are precisely the (set) Cartesian products $X \times Y$, where $X$ is an $S$-class of $G$ and $Y$ is an $S$-class of $H$. In other words, the vertices of $(G \times H) / S$ are $X \times Y$, where $X \in V(G / S)$ and $Y \in V(H / S)$. Further, there is a natural isomorphism

$$
\begin{array}{ccc}
(G \otimes H) / S & \longrightarrow & G / S \otimes H / S \\
X \times Y & \longmapsto & (X, Y) .
\end{array}
$$

In the proof our main theorem we will switch between $X \times Y$ and $(X, Y)$ when expedient.

The proof also uses all ideas discussed so far in this section.

Theorem 3.2. Suppose $G$ and $H$ are connected graphs. Then:

(1) $G$ or $H$ is even palindromic if and only if $G \otimes H$ is even palindromic.

(2) $G$ and $H$ are odd palindromic if and only if $G \otimes H$ is odd palindromic.

Proof. If $G$ or $H$ (say $G$ ) is even palindromic, then there exists an even palindromic involution $\alpha$ of $G$, so $(x, y) \mapsto(\alpha(x), y)$ is an even palindromic involution of $G \otimes H$. Next suppose $G$ and $H$ are odd palindromic. Then $G$ has an odd palindromic involution $\alpha$ with fixed point $x_{0}$, and $H$ has an odd palindromic involution $\beta$ with fixed point $y_{0}$. Then $(x, y) \mapsto(\alpha(x), \beta(y))$ is an odd palindromic involution of $G \otimes H$ whose sole fixed point is $\left(x_{0}, y_{0}\right)$.

It remains to prove the converses of the two statements. We will do this in three parts. The first part codifies the structure of involutions of $G \otimes H$.

Part I (Involution structure) Let $\varphi: G \otimes H \rightarrow G \otimes H$ be an involution. By the remarks preceding this theorem, $\varphi$ induces an automorphism $\widetilde{\varphi}$ of the $S$-thin graph $(G \otimes H) / S \cong$ $G / S \otimes H / S$. Because $\varphi$ is an involution, we have $\widetilde{\varphi}^{2}=$ id. (Note that $\widetilde{\varphi}$ could be the identity even if $\varphi$ is not. This is the case if $\varphi$ fixes each $S$-class, i.e., it restricts to a permutation on each $S$-class.) 
Take prime factorings $G / S=G_{1} \otimes \cdots \otimes G_{j}$ and $H / S=G_{j+1} \otimes \cdots \otimes G_{k}$. Then $\tilde{\varphi}$ is an automorphism (of order 1 or 2 ) of the graph

$$
G / S \otimes H / S=\left(G_{1} \otimes \cdots \otimes G_{j}\right) \otimes\left(G_{j+1} \otimes \cdots \otimes G_{k}\right) .
$$

Now, $\widetilde{\varphi}$ permutes the prime factors of this product in the sense of Theorem 3.1, where the permutation $\pi$ satisfies $\pi^{2}=\mathrm{id}$. As in the proof of Theorem 2.3, group together the prime factors $G_{i}$ of $G / S$ for which $1<\pi(i) \leq j$, and call their product $A$. Let $B$ be the product of the remaining factors of $G / S$. Also group together the prime factors $G_{i}$ of $H / S$ for which $j+1<\pi(i) \leq k$, and call their product $D$. The product of the remaining factors of $H / S$ is then a graph isomorphic to $B$. Now we have $G / S=A \otimes B$ and $H / S=B \otimes D$, and $\widetilde{\varphi}$ is an automorphism of

$$
G / S \otimes H / S=(A \otimes B) \otimes(B \otimes D)
$$

satisfying $\widetilde{\varphi}^{2}=\mathrm{id}$, and for which (as in the proof of Theorem 2.3) we have

$$
\widetilde{\varphi}\left((a, b),\left(b^{\prime}, d\right)\right)=\left(\left(\alpha(a), \beta\left(b^{\prime}\right)\right),\left(\beta^{-1}(b), \delta(d)\right)\right)
$$

for automorphisms $\alpha: A \rightarrow A, \beta: B \rightarrow B$ and $\delta: D \rightarrow D$, with $\alpha^{2}=$ id and $\delta^{2}=$ id.

In (3.3), the ordered pairs $(a, b)$ and $\left(\alpha(a), \beta\left(b^{\prime}\right)\right)$ are vertices of $G / S$, which are $S$ classes of $G$ (subsets of $V(G)$ ), and hence they have cardinalities $|(a, b)|$ and $\left|\left(\alpha(a), \beta\left(b^{\prime}\right)\right)\right|$. Similarly, $\left(b^{\prime}, d\right)$ and $\left(\beta^{-1}(b), \delta(d)\right)$ are $S$-classes of $H / S$.

By the remarks preceding this theorem, the involution $\varphi$ of $G \otimes H$ sends the $S$-class $(a, b) \times\left(b^{\prime}, d\right)$ bijectively to $S$-class $\left(\alpha(a), \beta\left(b^{\prime}\right)\right) \times\left(\beta^{-1}(b), \delta(d)\right)$, so

$$
|(a, b)| \cdot\left|\left(b^{\prime}, d\right)\right|=\left|\left(\alpha(a), \beta\left(b^{\prime}\right)\right)\right| \cdot\left|\left(\beta^{-1}(b), \delta(d)\right)\right|
$$

for all $a \in V(A), b, b^{\prime} \in V(B)$ and $d \in V(D)$. Putting $b^{\prime}=\beta^{-1}(b)$ yields

$$
\left.|(a, b)| \cdot\left|\left(\beta^{-1}(b), d\right)\right|=\mid(\alpha(a), b)\right)|\cdot|\left(\beta^{-1}(b), \delta(d)\right) \mid \cdot
$$

In (3.5) replace $d$ with $\delta(d)$ (and use $\delta^{2}=\mathrm{id}$ ) to get

$$
\left.|(a, b)| \cdot\left|\left(\beta^{-1}(b), \delta(d)\right)\right|=\mid(\alpha(a), b)\right)|\cdot|\left(\beta^{-1}(b), d\right) \mid \cdot
$$

Equations (3.5) and (3.6) imply $|(a, b)|=|(\alpha(a), b)|$. Form an automorphism $\widetilde{\alpha}$ : $A \otimes B \rightarrow A \otimes B$ as $\widetilde{\alpha}(a, b)=(\alpha(a), b))$. Then $\widetilde{\alpha}^{2}=$ id, so we have an involution (if it is not the identity map) $\widetilde{\alpha}: G / S \rightarrow G / S$ that maps each vertex ( $S$-class) $(a, b)$ to the vertex ( $S$-class) $(\alpha(a), b)$ of the same cardinality.

Also (3.5) and (3.6) yield $\left|\left(\beta^{-1}(b), \delta(d)\right)\right|=\left|\left(\beta^{-1}(b), d\right)\right|$, so $|(b, \delta(d))|=|(b, d)|$ for all $b \in V(B)$ and $d \in V(D)$. Form the automorphism $\widetilde{\delta}: B \otimes D \rightarrow B \otimes D$ where $\widetilde{\delta}(b, d)=(b, \delta(d))$. Then $\widetilde{\delta}^{2}=$ id, so we have an involution (if not the identity map) $\widetilde{\delta}$ : $H / S \rightarrow H / S$ mapping each $S$-class $(b, d)$ to the $S$-class $(b, \delta(d))$ of the same cardinality.

In summary, for any involution $\varphi$ of $G \otimes H$, we have constructed automorphisms $\widetilde{\alpha}$ and $\widetilde{\delta}$ of $G / S$ and $H / S$, respectively, for which $\widetilde{\alpha}^{2}=$ id and $\widetilde{\delta}^{2}=$ id. And $|\widetilde{\alpha}((a, b))|=|(a, b)|$ for any $S$-class $(a, b)$ of $G$. Thus we can lift $\widetilde{\alpha}$ to an automorphism $\lambda: G \rightarrow G$ by declaring that $\lambda$ restricts to a bijection $(a, b) \rightarrow(\alpha(a), b)$, for each $S$-class $(a, b)$ of $G$. Similarly, $|\widetilde{\delta}((b, d))|=|(b, d)|$ for any $S$-class $(b, d)$ of $H$, so we can lift $\widetilde{\delta}$ to an automorphism $\mu: H \rightarrow H$. In parts II and III of the proof these lifts will be palindromic involutions. 
To carry out this plan we will need to consider $S$-classes of $G \otimes H$ that are fixed by $\varphi$ (i.e., the $S$-classes whose vertices are permuted by $\varphi$.) By Equation (3.3), the fixed points of $\widetilde{\varphi}$ (respectively, the fixed $S$-classes of $\varphi$ ) are

$$
\begin{array}{ll}
\left(\left(a_{0}, \beta(b)\right),\left(b, d_{0}\right)\right) & \text { where } \alpha\left(a_{0}\right)=a_{0}, \delta\left(d_{0}\right)=d_{0} \text { and } b \in V(B) \\
\left(a_{0}, \beta(b)\right) \times\left(b, d_{0}\right) & \text { where } \alpha\left(a_{0}\right)=a_{0}, \delta\left(d_{0}\right)=d_{0} \text { and } b \in V(B) .
\end{array}
$$

We call an $S$-class even (odd) if it has even (odd) cardinality.

Part II (Converse of Statement (1)) Suppose $G \otimes H$ is even palindromic. Then there is an even palindromic involution $\varphi$ of $G \otimes H$. We retain the development and notation of Part I of the proof.

Our strategy is to show that one of $\widetilde{\alpha}: G / S \rightarrow G / S$ or $\widetilde{\delta}: H / S \rightarrow H / S$ has no odd fixed point ( $S$-class). For if this is the case for (say) $\widetilde{\alpha}$, then $\widetilde{\alpha}$ can be lifted to an automorphism $\lambda: G \rightarrow G$ sending any $S$-class $(a, b)$ bijectively to $(\alpha(a), b)$. Whenever $\widetilde{\alpha}$ fixes an $S$-class $(a, b)$, we can arrange for $\lambda$ to restrict to an order-2 fixedpoint-free permutation of the even set $(a, b)$. Then $\lambda$ will be an even palindromic involution of $G$, so $G$ is even palindromic.

Suppose to the contrary that $\widetilde{\alpha}$ had an odd fixed point $(a, b)$ and $\widetilde{\delta}$ had an odd fixed point $\left(b^{\prime}, d\right)$. (So $\alpha(a)=a$ and $\delta(d)=d$.) By (3.4),

$$
\underbrace{|(a, b)|}_{\text {odd }} \cdot \underbrace{\left|\left(b^{\prime}, d\right)\right|}_{\text {odd }}=\left|\left(a, \beta\left(b^{\prime}\right)\right)\right| \cdot\left|\left(\beta^{-1}(b), d\right)\right| .
$$

Then $\left(a, \beta\left(b^{\prime}\right)\right)$ is odd, so $\left(a, \beta\left(b^{\prime}\right)\right) \times\left(b^{\prime}, d\right)$ is an odd $S$-class of $G \otimes H$. But the involution $\varphi$ fixes this odd $S$-class, by (3.8). Thus $\varphi$ fixes some point of this $S$-class, contradicting the fact that $\varphi$ is even palindromic.

Part III (Converse of Statement (2)) Suppose $G \otimes H$ is odd palindromic. Then there is an odd palindromic involution $\varphi$ of $G \otimes H$ with fixed point $\left(x_{0}, y_{0}\right)$. Then $\varphi$ fixes the $S$-class $X$ that contains $\left(x_{0}, y_{0}\right)$, which necessarily has form $X=\left(a_{0}, \beta\left(b_{0}\right)\right) \times\left(b_{0}, d_{0}\right)$, where $\alpha\left(a_{0}\right)=a_{0}$ and $\delta\left(d_{0}\right)=d_{0}$. (See (3.8) in Part I.) As the involution $\varphi$ fixes exactly one vertex, which is in $X$, we know $X$ has odd cardinality. Thus $\left(a_{0}, \beta\left(b_{0}\right)\right)$ is an odd $S$-class of $G / S$, and $\left(b_{0}, d_{0}\right)$ is an odd $S$-class of $H / S$. Note that $\left(a_{0}, \beta\left(b_{0}\right)\right)$ is a fixed point of $\widetilde{\alpha}$ and $\left(b_{0}, d_{0}\right)$ is a fixed point of $\widetilde{\delta}$. Suppose $\widetilde{\delta}$ had another odd fixed point $\left(b_{1}, d_{1}\right)$. Then $\delta\left(d_{1}\right)=d_{1}$ and by Equation (3.4),

$$
\underbrace{\left|\left(a_{0}, \beta\left(b_{0}\right)\right)\right|}_{\text {odd }} \cdot \underbrace{\left|\left(b_{1}, d_{1}\right)\right|}_{\text {odd }}=\left|\left(a_{0}, \beta\left(b_{1}\right)\right)\right| \cdot\left|\left(b_{0}, d_{1}\right)\right| .
$$

Therefore $\left|\left(a_{0}, \beta\left(b_{1}\right)\right)\right|$ and $\left|\left(b_{0}, d_{1}\right)\right|$ are odd. Then $\left(a_{0}, \beta\left(b_{1}\right)\right) \times\left(b_{1}, d_{1}\right)$ and $\left(a_{0}, \beta\left(b_{0}\right)\right) \times$ $\left(b_{0}, d_{1}\right)$ are odd $S$-classes of $G \times H$ that are fixed by $\varphi$. But $X=\left(a_{0}, \beta\left(b_{0}\right)\right) \times\left(b_{0}, d_{0}\right)$ is the only such $S$-class, hence $\beta\left(b_{1}\right)=\beta\left(b_{0}\right)$ and $d_{1}=d_{0}$. This means $\left(b_{1}, d_{1}\right)=\left(b_{0}, d_{0}\right)$. Conclusion: $\left(b_{0}, d_{0}\right)$ is the only odd $S$-class of $H / S$ that is fixed by $\widetilde{\delta}$. Therefore we can lift $\widetilde{\delta}: H / S \rightarrow H / S$ to an odd palindromic involution $\mu: H \rightarrow H$ sending each $S$-class $(b, d)$ bijectively to $(b, \delta(d))$, having only one fixed vertex on the odd fixed class $\left(b_{0}, d_{0}\right)$ and no fixed points on any other fixed (even) $S$-class. Thus $H$ is odd palindromic.

By a symmetric argument, $G$ is also odd palindromic. 


\section{Conclusion and Open Questions}

Our Theorems 2.3 and 3.2 characterize palindromic Cartesian and strong products in terms of the palindromic properties of their factors. There are four standard associative graph products, the Cartesian, strong, direct and lexicographic products. (See [2].) Here we have only addressed two of these four products. A natural unexplored problem, then, is to establish analogous results for palindromic direct and lexicographic products. However, because the automorphism structure of these products is not as rigid as for the Cartesian and strong products (cf. Theorems 2.2 and 3.1 above), the results and proofs are likely to be substantially different from those presented here.

\section{ORCID iDs}

Richard H. Hammack (D) https://orcid.org/0000-0002-6384-9330

Jamie L. Shive (D) https://orcid.org/0000-0002-8294-1423

\section{References}

[1] R. A. Beeler, Palindromic graphs, Bull. Inst. Combin. Appl. 86 (2019), 85-100.

[2] R. Hammack, W. Imrich and S. Klavžar, Handbook of product graphs, Discrete Mathematics and its Applications (Boca Raton), CRC Press, Boca Raton, FL, 2nd edition, 2011, with a foreword by Peter Winkler.

[3] G. Sabidussi, Graph multiplication, Math. Z. 72 (1959/60), 446-457, doi:10.1007/bf01162967.

[4] V. G. Vizing, The cartesian product of graphs, Vyčisl. Sistemy No. 9 (1963), 30-43.

[5] D. B. West, Introduction to graph theory, Prentice Hall, Inc., Upper Saddle River, NJ, 1996. 